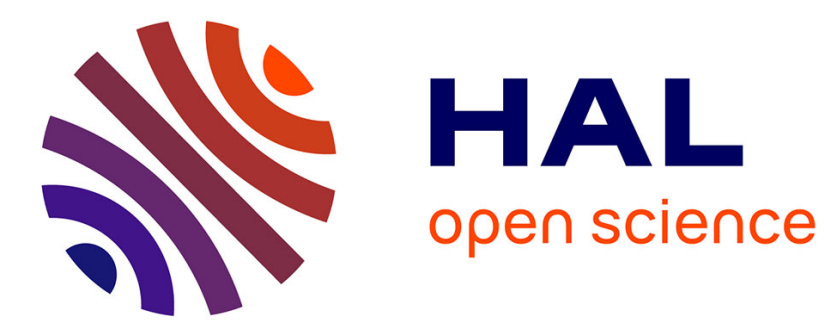

\title{
Suboptimal LMI-based Solution of Minimum Time Control Problem
}

\author{
Andrey Polyakov, Jean-Pierre Richard
}

\section{To cite this version:}

Andrey Polyakov, Jean-Pierre Richard. Suboptimal LMI-based Solution of Minimum Time Control Problem. IFAC World Congress, Aug 2014, Cape Town, South Africa. hal-00977875

\section{HAL Id: hal-00977875}

\section{https://hal.inria.fr/hal-00977875}

Submitted on 11 Apr 2014

HAL is a multi-disciplinary open access archive for the deposit and dissemination of scientific research documents, whether they are published or not. The documents may come from teaching and research institutions in France or abroad, or from public or private research centers.
L'archive ouverte pluridisciplinaire HAL, est destinée au dépôt et à la diffusion de documents scientifiques de niveau recherche, publiés ou non, émanant des établissements d'enseignement et de recherche français ou étrangers, des laboratoires publics ou privés. 


\title{
Suboptimal LMI-based Solution of Minimum Time Control Problem
}

\author{
A. Polyakov ${ }^{*, * *}$, J.-P. Richard ${ }^{* * * *}$ \\ * NON-A, INRIA - LNE, Parc Scientifique de la Haute Borne 40, \\ avenue Halley Bat.A, Park Plaza 59650 Villeneuve d'Ascq (e-mail: \\ andrey.polyakov@inria.fr). \\ ** LAGIS UMR CNRS 8219, Ecole Centrale de Lille, BP 48, Cité \\ Scientifique, 59651 Villeneuve-d'Ascq, \\ France.(e-mail:jean-pierre.richard@ec-lille.fr)
}

\begin{abstract}
The problem of time optimal control design is considered for a chain of integrators. The suboptimal solution based on Implicit Lyapunov Function (ILF) method is presented in the form of continuous stabilizing feedback. The Semi-Definite Programming (SDP) problem with the constraints in the form of Linear Matrix Inequalities (LMI) is obtained for tuning the optimal parameters. The suboptimal solution is compared with the minimum discontinuous feedback on numerical example.
\end{abstract}

\section{INTRODUCTION}

The control applications frequently asks for algorithms, which provide terminations of transition processes in a minimal possible time. Such problem statements usually appear in robotic systems, aerospace applications, underwater/surface vehicles control systems, etc. Despite of long history of the optimal control theory (Pontryagin et al. [1962], Bellman [1957]) time optimal control problems are still subjects of intensive researches, see for example, Lasserre et al. [2005], Chernousko et al. [2008], Dinuzzo and Ferrara [2009], Boltyanski and Poznyak [2012].

The "bang-bang" control is usual solution of the minimum time control problem. Sometimes, continuous control strategies are preferable in practice, see, for example, Bernstein [1995], Glizer and Turetsky [2012]. Suboptimal robust control solutions have a great practical interest (Bertsekas [2005], Bohl and McAvoy [1976]), since they are usually obtained under additional restrictions to the control forms, which are required for a practical control application and robustness of the control strategy.

The present paper considers the problem of time optimal control synthesis for a chain of integrators controlled by linear dynamic feedbacks. The design concept is based on the Implicit Lyapunov Function (ILF) method (Korobov [1979], Adamy and Flemming [2004], Polyakov et al. [2013]) in order to present the suboptimal solution for minimum time control problem. This method uses Lyapunov function defined in the implicit form by means of an algebraic equation. Convergence and stability analysis in this case does not require solving of this equation, since the Implicit function theorem (see, for example, Courant and John [2000]) helps to provide all required analysis using the algebraic equation only. The ILF control design scheme has representation in the form of Linear Matrix Inequalities (LMI), see Polyakov et al. [2013]. The time optimization problem in this case becomes SDP (semidefinite programming) formalization.
The paper is organized as follows. The next section introduces some notations used in the paper. The section 3 presents the problem statement. The section 4 gives some preliminary remarks about finite-time ILF method. Then the control design schemes are given and studied. The aspects of the practical implementation of the developed control schemes are discussed in the section 6 . Finally, numerical examples and conclusions are presented.

\section{NOTATION}

Through the paper the following notations will be used:

- $\mathbb{R}_{+}=\{x \in \mathbb{R}: x>0\}, \mathbb{R}_{-}=\{x \in \mathbb{R}: x<0\}$, where $\mathbb{R}$ is the set of real number;

- $i=\sqrt{-1}$ is the imaginary unit;

- for a differential equation numbered as (.), the time derivative of a function $V$ along the solution of (.) is denoted by $\left.\frac{d V}{d t}\right|_{(.)}$;

- $\|\cdot\|$ is the Euclidian norm in $\mathbb{R}^{n}$, i.e. $\|x\|=$ $\sqrt{x_{1}^{2}+\ldots+x_{n}^{2}}$ for $x=\left(x_{1}, \ldots, x_{n}\right)^{T} \in \mathbb{R}^{n}$

- $\operatorname{diag}\left\{\lambda_{i}\right\}_{i=1}^{n}$ is the diagonal matrix with the elements $\lambda_{i}$ on the main diagonal;

- a continuous function $\sigma: \mathbb{R}_{+} \rightarrow \mathbb{R}_{+}$belongs to the class $\mathcal{K}$ if it is monotone increasing and $\sigma(0)=0$;

- for a matrix $P \in \mathbb{R}^{n \times n}$, which has the real spectrum, the minimal and maximal eigenvalues are denoted by $\lambda_{\min }(P)$ and $\lambda_{\max }(P)$, respectively;

- if $P \in \mathbb{R}^{n \times n}$ then the inequality $P>0(P \geq$ $0, P<0, P \leq 0)$ means that $P$ is symmetric and positive definite (positive semidefinite, negative definite, negative semidefinite).

- $\mathbb{C}_{[a, b]}^{k}$ is a set of functions defined on $[a, b]$, which are continuously differentiable at least up to the order $k$.

- $\mathcal{L}_{[a, b]}$ is a set of Lebesgue integrable functions defined on $[a, b]$.

\section{PROBLEM STATEMENT}

Let us consider a single input control system of the form 


$$
\dot{x}=A x+b u, \quad t \in[0, T], \quad x(0)=x_{0} \in \mathbb{R}^{n},
$$

where $T \in R_{+}$is a finite instant of time, $x \in \mathbb{R}^{n}$ is the state vector, $u \in \mathbb{R}$ is a control input,

$$
A=\left(\begin{array}{ccccc}
0 & 1 & 0 & \ldots & 0 \\
0 & 0 & 1 & \ldots & 0 \\
\ldots & \ldots & \ldots & \ldots & \ldots \\
0 & 0 & 0 & \ldots & 1 \\
0 & 0 & 0 & \ldots & 0
\end{array}\right) \quad \text { and } \quad b=\left(\begin{array}{c}
0 \\
0 \\
\ldots \\
0 \\
1
\end{array}\right)
$$

The system (1) describes a controlled chain of integrators. The model of the control system like (1) is motivated by many mechanical and electromechanical applications, see, for example, Chernousko et al. [2008], Utkin et al. [2009], Biagiotti and Zanasi [2010].

Let us consider the time-optimal control problem

$$
T \rightarrow \min
$$

subject to

$$
\begin{gathered}
u(\cdot) \in\left\{u(\cdot) \in \mathcal{L}_{[0, T]}:|u(t)| \leq u_{0}, t \in[0, T]\right\}, \\
x(\cdot) \in \mathbb{C}_{[0, T]}^{1}:\left\{\begin{array}{l}
\dot{x}(t)=A x(t)+b u(t), \\
x(0)=x_{0}, \quad x(T)=0,
\end{array}\right.
\end{gathered}
$$

where $x_{0} \in \mathbb{R}^{n}$ and $u_{0} \in \mathbb{R}_{+}$are given.

The optimization problem (2), (3) is the classical timeoptimal control problem. According to the celebrated theorem by Feldbaum [1953] the optimal solution of this problem is the so-called bang-bang control, which is a piecewise constant function $u_{\text {opt }}(t) \in\left\{-u_{0}, u_{0}\right\}$ with $n$ switching instants (including $t=0$ ). This paper addresses the suboptimal control design to the optimization problem (2), (3) under some additional assumptions. The first one is the following:

$$
\begin{gathered}
u(\cdot) \in \mathcal{U}, \\
\mathcal{U}=\left\{u(\cdot) \in \mathbb{C}_{[0, T]} \cap \mathbb{C}_{[0, T)}^{\infty}:|u(t)| \leq u_{0}, t \in[0, T]\right\}
\end{gathered}
$$

where $u_{0} \in \mathbb{R}_{+}$is some given number. In other words, the scalar control input $u \in \mathbb{R}$ is assumed to be bounded and smooth.

The saturation and smoothness of the input signal are the natural practical restrictions of control systems considered in many papers, see for example, Wang et al. [2012], Tarbouriech and Garcia [1997]. Frequently, these conditions are necessary for a physical realization of a control algorithm.

Since the linear feedback strategies may provide a nearly time-optimal solutions (Bohl and McAvoy [1976]), then let us restrict additionally admissible control strategies to the class of linear time-varying feedbacks.

The main aim of this paper is to propose an algorithm for time suboptimal control design

$$
\begin{gathered}
u(\cdot)=w^{T}(\cdot) x(\cdot) \in \mathcal{U}, \\
w=\left(w_{1}, w_{2}, \ldots, w_{n}\right)^{T} \in \mathbb{R}^{n}, \quad w_{i}(\cdot) \in \mathbb{C}_{[0, T)}^{\infty},
\end{gathered}
$$

which admits the simple scheme of parameters tuning based on LMI formalism. Such approach allows us to reduce (relax) the infinite dimensional optimization problem (2), (3) to a finite dimensional one, which provides a suboptimal solution.

The last additional assumption is not constructive from the mathematical point of view. However, it is very impor- tant for control practice, which frequently looks for simple solutions for improvement of the control performance.

Another problem, which is discussed in the paper, is the robustness of the proposed control scheme. Robustification of the optimal solutions is a very important task (Boltyanski and Poznyak [2012], Chernousko et al. [2008], Dinuzzo and Ferrara [2009]).

\section{FINITE-TIME ANALYSIS USING IMPLICIT LYAPUNOV FUNCTION}

Consider the system of the form

$$
\dot{x}=f(t, x), \quad x(0)=x_{0},
$$

where $x \in \mathbb{R}^{n}$ is the state vector, $f: \mathbb{R}_{+} \times \mathbb{R}^{n} \rightarrow \mathbb{R}^{n}$ is a continuous nonlinear vector field, which is locally Lipschitz outside the origin.

Assume that the origin is an equilibrium point of the system (6), i.e. $f(t, 0)=0$.

Definition 1. (Roxin [1966], Bhat and Bernstein [2000]).

The origin of the system (6) is said to be finite-time stable if for a set $\mathcal{V} \subset \mathbb{R}^{n}$ :

(1) Finite-time attractivity: there exists a function $T$ : $\mathcal{V} \backslash\{0\} \rightarrow \mathbb{R}_{+}$, such that for all $x_{0} \in \mathcal{V} \backslash\{0\}, x\left(t, x_{0}\right)$ is defined on $\left[0, T\left(x_{0}\right)\right)$ and $\lim _{t \rightarrow T\left(x_{0}\right)} x\left(t, x_{0}\right)=0$.

(2) Lyapunov stability: there exists a function $\delta \in \mathcal{K}$ such that for all $x_{0} \in \mathcal{V},\left\|x\left(t, x_{0}\right)\right\| \leq \delta\left(\left\|x_{0}\right\|\right)$.

The function $T(\cdot)$ from Definition 1 is called the settlingtime function of the system (6).

If the set $\mathcal{V}$ in Definition 1 coincides with $\mathbb{R}^{n}$ then the origin of the system (6) is globally finite time stable.

The next theorem presents the extension of the ILF method Adamy and Flemming [2004] for finite-time stability analysis.

Theorem 2. (Polyakov et al. [2013]). If there exists a continuous function

$$
\begin{gathered}
Q: \mathbb{R}_{+} \times \mathbb{R}^{n} \rightarrow \mathbb{R} \\
(V, x) \mapsto Q(V, x)
\end{gathered}
$$

such that

C1) $Q$ is continuously differentiable $\forall x \in \mathbb{R}^{n} \backslash\{0\}$ and $\forall V \in \mathbb{R}_{+}$;

C2) for any $x \in \mathbb{R}^{n} \backslash\{0\}$ there exist $V^{-} \in \mathbb{R}_{+}$and $V^{+} \in \mathbb{R}_{+}: Q\left(V^{-}, x\right)<0<Q\left(V^{+}, x\right)$;

C3) $\lim _{\substack{x \rightarrow 0 \\(V, x) \in \Omega}} V=0^{+}, \quad \lim _{\substack{V \rightarrow 0^{+} \\(V, x) \in \Omega}}\|x\|=0, \quad \lim _{\substack{\|x\| \rightarrow \infty \\(V, x) \in \Omega}} V=+\infty$, where $\Omega=\left\{(V, x) \in \mathbb{R}^{n+1}: Q(V, x)=0\right\}$;

$C 4)$ the inequality $-\infty<\frac{\partial Q(V, x)}{\partial V}<0$ holds $\forall V \in \mathbb{R}_{+}$and $\forall x \in \mathbb{R}^{n} \backslash\{0\}$

$C 5)$ for $\forall t \in \mathbb{R}_{+}$and $\forall(V, x) \in \Omega$;

$$
\frac{\partial Q(V, x)}{\partial x} f(t, x) \leq c V^{1-\mu} \frac{\partial Q(V, x)}{\partial V}
$$

where $c>0$ and $0<\mu \leq 1$ are some constants, then the origin of system (6) is globally finite-time stable with the settling-time estimate $T\left(x_{0}\right) \leq \frac{V_{0}^{\mu}}{c \mu}$, where $V_{0} \in \mathbb{R}_{+}$: $Q\left(V_{0}, x_{0}\right)=0$. 
The ILF method defines a Lyapunov function $V$ for the a system in the implicit form, for instance, as a solution of the algebraic equation $Q(V, x)=0$. Conditions $C 1)$ $C 4$ ) of Theorem 2 guarantee the properness (positive definiteness, radial unboundedness) of the corresponding solution. Due to the classical implicit function theorem (see, for example, Courant and John [2000]) the partial derivative of the Lyapunov function $V$ can be calculated as follows $\frac{\partial V}{\partial x}=-\left[\frac{\partial Q}{\partial V}\right]^{-1} \frac{\partial Q}{\partial x}$. Therefore, the condition $\left.C 5\right)$ guarantees negative definiteness of the total derivative of $V$ and finite-time stability of the system (6).

\section{CONTROL DESIGN}

\subsection{Finite-Time Control}

Introduce the Implicit Lyapunov Function

$$
Q(V, x):=x^{\top} D_{\mu}\left(V^{-1}\right) P D_{\mu}\left(V^{-1}\right) x-1,
$$

where $D_{\mu}(\lambda)$ is the dilation matrix of the form

$$
D_{\mu}(\lambda)=\operatorname{diag}\left\{\lambda^{1+(n-i) \mu}\right\}_{i=1}^{n}, \quad 0<\mu \leq 1
$$

and $P \in \mathbb{R}^{n \times n}$ is a symmetric positive definite matrix, i.e. $P=P^{\top}>0$.

The following Theorem presents the main scheme of the ILF control design. It refines the result of Polyakov et al. [2013] providing the precise estimate of the settling time. Denote $H_{\mu}:=\operatorname{diag}\{1+(n-i) \mu\}_{i=1}^{n}$.

Theorem 3. Let $X \in \mathbb{R}^{n \times n}$ and $y \in \mathbb{R}^{1 \times n}$ satisfy the following system of LMIs:

$$
\left\{\begin{array}{c}
A X+X A^{\top}+b y+y^{\top} b^{\top}+H_{\mu} X+X H_{\mu}=0, \\
X H_{\mu}+H_{\mu} X>0, \quad X>0
\end{array}\right.
$$

for some $\mu \in(0,1]$. Then the control of the form

where $k=y X^{-1}$,

$$
u(V, x)=V^{1-\mu} k D_{\mu}\left(V^{-1}\right) x,
$$

$$
V \in \mathbb{R}_{+}: Q(V, x)=0
$$

and $Q(V, x)$ is defined by (7) with $P=X^{-1}$, stabilizes the system (1) in a finite time with settling-time function defined as follows

$$
T\left(x_{0}\right)=\frac{V_{0}^{\mu}}{\mu},
$$

where $V_{0} \in \mathbb{R}_{+}: Q\left(V_{0}, x_{0}\right)=0$.

Proof. The function $Q(V, x)$ defined by (7) satisfies the conditions C1)-C3) of Theorem 2. Indeed, it is continuously differentiable for all $V \in \mathbb{R}_{+}$and $\forall x \in \mathbb{R}^{n}$. Since $P>0$ then the following chain of inequalities

$$
\frac{\lambda_{\min }(P)\|x\|^{2}}{\max \left\{V^{1+(n-1) \mu}, V\right\}} \leq Q(V, x)+1 \leq \frac{\lambda_{\max }(P)\|x\|^{2}}{\min \left\{V^{1+(n-1) \mu}, V\right\}}
$$

implies that for any $x \in \mathbb{R}^{n} \backslash\{0\}$ there exist $V^{-} \in \mathbb{R}_{+}$ and $V^{+} \in \mathbb{R}_{+}: Q\left(V^{-}, x\right)<0<Q\left(V^{+}, x\right)$. Moreover, if $Q(V, x)=0$ then the same chain of inequalities gives

$$
\frac{\min \left\{V^{1+(n-1) \mu}, V\right\}}{\lambda_{\max }(P)} \leq\|x\|^{2} \leq \frac{\max \left\{V^{1+(n-1) \mu}, V\right\}}{\lambda_{\min }(P)} .
$$

It follows that the condition C3) of Theorem 2 holds.

Since

$$
\frac{\partial Q}{\partial V}=-V^{-1} x^{\top} D_{\mu}\left(V^{-1}\right)\left(H_{\mu} P+P H_{\mu}\right) D_{\mu}\left(V^{-1}\right) x
$$

then (8) and $P:=X^{-1}$ implies $H_{\mu} P+P H_{\mu}>0$ and $\frac{\partial Q}{\partial V}<0$ for $\forall V \in \mathbb{R}_{+}$and $x \in \mathbb{R}^{n} \backslash\{0\}$. So the condition $C_{4}$ ) of Theorem 2 also holds. In this case we have

$\frac{\partial Q}{\partial x}(A x+b u(x))=2 x^{\top} D_{r}\left(V^{-1}\right) P D_{\mu}\left(V^{-1}\right)(A x+b u(x))$.

Taking into account that $D_{r}\left(V^{-1}\right) A D_{r}^{-1}\left(V^{-1}\right)=V^{-\mu} A$ and $D_{\mu}\left(V^{-1}\right) b u(x)=V^{-\mu} b k D_{\mu}\left(V^{-1}\right) x$ we obtain

$$
\begin{gathered}
\frac{\partial Q}{\partial x}(A x+b u(x))= \\
V^{-\mu} x^{\top} D_{\mu}\left(V^{-1}\right)\left(P(A+b k)+(A+b k)^{\top} P\right) D_{\mu}\left(V^{-1}\right) x
\end{gathered}
$$

Therefore

$$
\begin{gathered}
\left.\frac{d V}{d t}\right|_{(1)}=-\left[\frac{\partial Q}{\partial V}\right]^{-1} \frac{\partial Q}{\partial x}(A x+b u(x))= \\
\frac{x^{\top} D_{\mu}\left(V^{-1}\right)\left(P(A+b k)+(A+b k)^{\top} P\right) D_{\mu}\left(V^{-1}\right) x}{x^{\top} D_{\mu}\left(V^{-1}\right)\left(H_{\mu} P+P H_{\mu}\right) D_{\mu}\left(V^{-1}\right) x} V^{1-\mu} \\
=\frac{z^{\top}\left(P(A+b k)+(A+b k)^{\top} P\right) z}{z^{\top}\left(H_{\mu} P+P H_{\mu}\right) z} V^{1-\mu}=-V^{1-\mu}
\end{gathered}
$$

where $z:=z(V, x)=D_{\mu}\left(V^{-1}\right) x$. The last equality implies the finite-time stability of the system (1) and the representation (10) for the settling-time function.

The main advantage of the presented control scheme is the LMI representation of the procedure for parameters tuning. Any existing LMI solver can be used in order to select the control parameters.

Proposition 4. (Polyakov et al. [2014]). The LMI system (8) is feasible for all $\mu>0$.

A practical implementation of the control (9) admits a simple realization if the initial state $x(0)=x_{0} \in \mathbb{R}^{n}$ of the system (1) is given. Indeed, since the function $Q\left(V_{0}, x_{0}\right)$ satisfies the conditions of Theorem 2 then the equation $Q\left(V_{0}, x_{0}\right)=0$ has a unique positive solution $V_{0} \in \mathbb{R}_{+}$ for any given $x_{0} \in \mathbb{R}^{n}$. The corresponding solution can be found numerically using, for example, the simplest bisection method.

Since the function $V$ is a solution of (see the proof of Theorem 3) the Cauchy problem $\dot{V}(t)=-V^{1-\mu}, V(0)=$ $V_{0}$, then it can be found analytically

$$
V(t)=\left(V_{0}^{\mu}-\mu t\right)^{1 / \mu}, \quad t \in\left[0, V_{0}^{\mu} / \mu\right] .
$$

If the linear dynamic feedback $u(\cdot)$ has the form (9) with $V=V(t)$, where $V(t)$ is defined by (11) with $V_{0}$ satisfying the equation $Q\left(V_{0}, x_{0}\right)=0$ for a given $x_{0} \in \mathbb{R}^{n}$, then the condition $x\left(V_{0}^{\mu} / \mu\right)=0$ fulfills on the corresponding trajectory of the closed system (1).

\subsection{Suboptimal Finite-Time Control}

The value $T=V_{0}^{\mu} / \mu$ has to be minimized under the constraint $u(\cdot) \in \mathcal{U}$, where $u$ is defined by (9), in order to obtain a suboptimal solution to the problem (2), (3). Let us formulate a finite dimensional optimization problem with the LMI constraints, which provides the required suboptimal solution.

Corollary 5. If for some $\mu \in(0,1], x_{0} \in \mathbb{R}^{n} \backslash\{0\}$ and $T \in \mathbb{R}_{+}$the LMI system (8) is feasible together with

$$
\left(\begin{array}{cc}
1 & x_{0}^{\top} \\
x_{0} & D_{\mu}\left((\mu T)^{1 / \mu}\right) X D_{\mu}\left((\mu T)^{1 / \mu}\right)
\end{array}\right) \geq 0
$$


where $X \in \mathbb{R}^{n \times n}$ and $y \in \mathbb{R}^{1 \times n}$, then the ILF control (9) guarantees that the settling time function $T\left(x_{0}\right)$ of the system (1) satisfies the inequality $T\left(x_{0}\right) \leq T$, where $x_{0} \in \mathbb{R}^{n}$ is a given initial state.

Proof. Denote $V_{0} \in \mathbb{R}_{+}: Q\left(V_{0}, x_{0}\right)=0$.

The LMI (12) implies

$$
\begin{gathered}
Q\left((\mu T)^{1 / \mu}, x_{0}\right)= \\
x_{0}^{\top} D_{\mu}\left(\frac{1}{(\mu T)^{1 / \mu}}\right) X^{-1} D_{\mu}\left(\frac{1}{(\mu T)^{1 / \mu}}\right) x_{0}-1 \leq 0=Q\left(V_{0}, x_{0}\right) .
\end{gathered}
$$
Since $\frac{\partial Q(V, x)}{\partial V}<0$ for any $x \in \mathbb{R}^{n} \backslash\{0\}$ then $V_{0} \leq(\mu T)^{1 / \mu}$. According to Theorem 3 we obtain $T\left(x_{0}\right)=\frac{V_{0}^{\mu}}{\mu} \leq T$.

Minimization of the parameter $T \in \mathbb{R}_{+}$under the LMI constraints (8), (12) minimizes the settling time of the closed-loop system (1),(9). If the LMI (8) is feasible then the settling time $T\left(x_{0}\right)$ can be made infinitely small if the magnitude of the control signal is not bounded. Indeed, let the $\left(y_{0}, X_{0}\right)$ satisfies the LMI (8), then $y=\alpha y_{0}, X=\alpha X_{0}$ is also satisfies (8) for any $\alpha \in \mathbb{R}_{+}$. Since $X_{0}>0$ then for any $T>0$ and any $x_{0} \in \mathbb{R}^{n} \backslash\{0\}$ there exists $\alpha \in \mathbb{R}_{+}$such that the LMI (12) holds.

Corollary 6. Let for some $\mu \in(0,1], T \in \mathbb{R}_{+}$and $u_{0} \in \mathbb{R}_{+}$ the LMI system (8), (12) is feasible together with

$$
\left(\begin{array}{cc}
X & y^{\top} \\
y & \frac{u_{0}^{2}}{(\mu T)^{\frac{2-2 \mu}{\mu}}}
\end{array}\right) \geq 0
$$

where $X \in \mathbb{R}^{n \times n}$ and $y \in \mathbb{R}^{1 \times n}$. Then the ILF control (9) is bounded by $u_{0}$ along the generated trajectory $x(t)$ of the closed-loop system, i.e.

$$
|u(V(t), x(t))| \leq u_{0} \quad \forall t \in\left[0, T\left(x_{0}\right)\right] .
$$

Proof. Using Shur complement the LMI (13) can be rewritten in the form $\frac{(\mu T)^{\frac{2-2 \mu}{\mu}}}{u_{0}^{2}} y^{\top} y \leq X$. Taking into account $X=P^{-1}$ and $y=k P^{-1}$ we obtain $(\mu T)^{\frac{2-2 \mu}{\mu}} k^{\top} k \leq$ $u_{0}^{2} P$ and $\forall x \in \mathbb{R}^{n} \backslash\{0\}, \forall V \in \mathbb{R}_{+}$

$$
\begin{gathered}
(\mu T)^{\frac{2-2 \mu}{\mu}} x^{\top} D_{\mu}\left(V^{-1}\right) k^{\top} k D_{\mu}\left(V^{-1}\right) x \\
\leq u_{0}^{2} x^{\top} D_{\mu}\left(V^{-1}\right) P D_{\mu}\left(V^{-1}\right) x .
\end{gathered}
$$

On the one hand, since $V(t) \leq V_{0}$ for $t \in\left[0, T\left(x_{0}\right)\right]$ (see, formula (11)) and $T\left(x_{0}\right) \leq T$ then

$$
\begin{gathered}
u^{2}(t)=V^{2-2 \mu}(t) x^{\top}(t) D_{\mu}\left(V^{-1}(t)\right) k^{\top} k D_{\mu}\left(V^{-1}(t)\right) x(t) \\
\leq V_{0}^{2-2 \mu} x^{\top}(t) D_{\mu}\left(V^{-1}(t)\right) k^{\top} k D_{\mu}\left(V^{-1}(t)\right) x(t) \leq \\
(\mu T)^{\frac{2-2 \mu}{\mu}} x^{\top} D_{\mu}\left(V^{-1}(t)\right) k^{\top} k D_{\mu}\left(V^{-1}(t)\right) x .
\end{gathered}
$$

On the other hand, since $Q(V, x)=0$ then

$$
u_{0}^{2} x^{\top} D_{\mu}\left(V^{-1}\right) P D_{\mu}\left(V^{-1}\right) x=u_{0}^{2} .
$$

Therefore, $u^{2}(V(t), x(t)) \leq u_{0}^{2}$ for $t \in\left[0, T\left(x_{0}\right)\right]$.

The proven corollary allows designing the ILF control of the given maximum magnitude. If $\mu=1$ then the inequality (13) becomes independent of $T$ and the control is globally bounded. It is important to remark that the design procedure is again formulated in the LMI form.

For any fixed $\mu$ and $T$ the matrix inequalities (8), (12), (13) become LMIs, which can be easily solved using any LMI solver (for example, SeDuMi).
Summarizing the obtained results we can formulate the following finite dimensional optimization problem for a fixed $\mu \in(0,1]$ :

$$
T \rightarrow \min _{X \in \mathbb{R}^{n}, y \in \mathbb{R}^{1 \times n}}
$$

subject to $(8),(12),(13)$.

For the fixed parameter $\mu$ the presented optimization problem can be easily solved using, for example, the method of intervals (see, for example, Tuan et al. [1999]). Minimization with respect to $\mu$ can be done applying any derivative free method (for example, the function fminsearch of MATLAB).

\section{ON ROBUST DIGITAL IMPLEMENTATION OF SUBOPTIMAL ILF CONTROL}

As it was remarked above the value of the Lyapunov function can be calculated along the trajectory as follows $V(t)=\left(V_{0}^{\mu}-\mu t\right)^{1 / \mu}, \quad t \in\left[0, V_{0}^{\mu} / \mu\right]$. The suboptimal linear dynamic feedback $u(\cdot)$ can be defined in the form (9) with $V=V(t)$, where $V(t)$ is defined by (11) and by the equation $Q\left(V_{0}, x_{0}\right)=0$ for a given $x_{0} \in \mathbb{R}^{n}$. In this case the trajectory of the closed system (1) satisfies the condition $x\left(V_{0}^{\mu} / \mu\right)=0$.

In practice the original system (1) has some disturbances and uncertainties that can reduce the quality of the presented dynamic control. Therefore, some practical robustification procedures are required for the suboptimal ILF control scheme.

\subsection{Sampled-time realization of the ILF control}

The ideas of the sampled-time realization of the ILF control are discussed in Polyakov et al. [2013]. They are mainly based on two following corollaries, which can also be proven for the ILF control defined in Theorem 3.

Corollary 7. (Polyakov et al. [2013]) Let the control $u(V, x)$ be defined according to Theorem 3 then the control $u_{0}(x)=u\left(V_{0}, x\right)$ is the linear stabilizing feedback control for the system (1) for any given $V_{0} \in \mathbb{R}_{+}$.

The next corollary will help us to analyze the discrete-time version of the developed control schemes.

Corollary 8. (Polyakov et al. [2013]) Let $\left\{t_{i}\right\}_{i=0}^{\infty}$ be an arbitrary strictly increasing sequence of time instants, $0=t_{0}<t_{1}<t_{2}<\ldots$. Let the function $u(V, x)$ is defined according to Theorem 3 then the origin of the system (1) with the switching control

$$
u(x):=u\left(V_{i}, x\right) \quad \text { for } \quad t \in\left[t_{i}, t_{i+1}\right),
$$

where $V_{i}>0: Q\left(V_{i}, x\left(t_{i}\right)\right)=0$, is asymptotically stable.

The last corollary provides that the sampled-time realization of the ILF control keeps the stability property of the closed-loop system (1) independently on the sampling period.

Let the control $u(V, x)$ of the form (9) be realized in a digital device and the parameter $V$ may change its value at some time instants: $t_{0}=0, t_{i}>0, i=1,2, \ldots$. Denote $V_{i}:=V\left(t_{i}\right)$ and $x_{i}:=x\left(t_{i}\right)$. On the time interval $\left[t_{i}, t_{i+1}\right)$ the control has the form $u\left(V_{i}, x\right)$, which is a linear stabilizing feedback for any $V_{i} \in \mathbb{R}_{+}$(see, Corollary 7 ). 
The simplest scheme for selection of the switching control parameter $V_{i}$ is described by the following algorithm.

A L G O R I T H M (Polyakov et al. [2013])

INITIALIZATION:

STEP :

$$
V_{0}=1 ; a=V_{\min } ; b=1 ;
$$

$$
\begin{aligned}
& \text { If } x_{i}^{T} D_{\mu}\left(b^{-1}\right) P D_{\mu}\left(b^{-1}\right) x_{i}>1 \text { then } \\
& \quad a=b ; b=2 b ; \\
& \text { elseif } x_{i}^{T} D_{\mu}\left(a^{-1}\right) P D_{\mu}\left(a^{-1}\right) x_{i}<1 \text { then } \\
& \quad b=a ; a=\max \left\{\frac{a}{2}, V_{\min }\right\} ; \\
& \text { else } \quad c=\frac{a+b}{2} ; \\
& \quad \text { If } x_{i}^{T} D_{\mu}\left(c^{-1}\right) P D_{\mu}\left(c^{-1}\right) x_{i}<1 \text { then } b=c \text {; } \\
& \quad \text { else } a=\max \left\{V_{\min }, c\right\} ; \\
& \quad \text { endif; }
\end{aligned}
$$

endif ;

$V_{i}=b$;

Remark, STEP of Algorithm 6.1 can be realized just once in each sampled time instant.

The parameter $V_{\min }$ defines lower possible value of $V$. In practice, this parameter cannot be selected arbitrary small due to finite numerical precision of digital devices.

\subsection{Adaptive scheme}

An adaptive scheme can also be presented for practical implementation of ILF control.

Theorem 3 proves the following dynamic equation for the ILF $\dot{V}=-V^{1-\mu}$.

If this equation is calculated on-line with the initial condition $V(0)=V_{0}>0: x(0)^{T} D_{\mu}\left(V_{0}^{-1}\right) P D_{\mu}\left(V_{0}^{-1}\right) x(0)=1$ then the corresponding trajectory $x(t)$ of the system (1) with the control $\tilde{u}(V(t), x(t))$ converges to the origin in a finite time (see, Theorem 2 and the previous section).

In order to provide some robustness to the presented control scheme the dynamic equation $\dot{V}=-V^{1-\mu}$ can be modified as follows

$$
\dot{V}(t)=-H[-Q(V(t), x(t))] V^{1-\mu}(t),
$$

where $H[\rho]$ is a Heaviside step function

$$
H[\rho]=\left\{\begin{array}{l}
1 \text { for } \rho \geq 0 \\
0 \text { for } \rho<0 .
\end{array}\right.
$$

The modified dynamic equation (16) guarantees that the function $V(t)$ will be decreasing only in the case $x^{T}(t) D_{r}\left(V^{-1}(t)\right) P D_{r}\left(V^{-1}(t)\right) x(t) \leq 1$, i.e. if $s$ belongs to the ellipsoidal level set of the ILF. Otherwise, $\dot{V}=0$ and the function $V(t)$ is constant. Since $\tilde{u}(V, s)$ is a linear stabilizing feedback for any fixed $V>0$ then the adaptive scheme (16) of the ILF implementation will guarantee at least asymptotic stabilization of the system (1). For the chattering reduction the function $V(t)$ can also be restricted from below by some minimal value $V_{\min }$.

The Heaviside function in the equation (16) can be replaced with sign $[-Q(V(t), x(t)]$ providing additional computational robustness of the digital ILF control application. A more detailed study of the ILF control implementation goes out of the scope of this paper. It is suggested as a topic for future research. The numerical simulations given below demonstrate an effectiveness of the presented simplest algorithms of ILF control implementation.

\section{NUMERICAL EXAMPLE}

Consider the system (1) for $n=2$ under control restriction $|u| \leq 1$.

It is well known, see, for example, Chernousko et al. [2008], that the time optimal feedback law for this case has the discontinuous form

$$
u_{\text {opt }}\left(x_{1}, x_{2}\right)=-\operatorname{sign}\left(x_{2}+\sqrt{2\left|x_{1}\right|} \operatorname{sign}\left[x_{1}\right]\right) .
$$

The continuous stabilizing feedback (9) designed following the optimization procedure (14) for $\mu=1$ and $x_{0}=(1,0)$ has the parameters

$$
P=\left(\begin{array}{cc}
49.6139 & 18.8965 \\
18.8965 & 9.4482
\end{array}\right), k=\left(\begin{array}{ll}
-5.2511 & -3.0000
\end{array}\right) \text {. }
$$

The numerical solving of ODE for the closed-loop system have been done by the Euler method with the fixed step size and the discrete time application of the finite time ILF control is realized by the scheme presented in Algorithm 6.1. The adaptive scheme has also been verified by numerical simulation. It provides very similar results.

The results of numerical simulations for $h=0.001$ and for $V_{\min }=0.001$ are shown on Fig. 1 . It was expectable that the optimal controller provides the faster transition. However, if the control will be applied with larger sampling period, which frequently takes a place in real applications, then the situation is changing. Fig. 2 depicts the simulation results for $h=0.05$ and $V_{\min }=0.05$. Such defect of the optimal controller is known as "chattering phenomenon" (see, Utkin et al. [2009]), which appears in time-optimal solutions realized by sliding mode control feedbacks (Dinuzzo and Ferrara [2009]).

\section{CONCLUSIONS}

In the paper a new suboptimal control algorithm is presented. It has the following advantages:

- The control design algorithm is based on solving of SDP with LMI constrains.

- The suboptimal control algorithm admits several schemes for robust practical realization: time-varying linear feedback, linear switching feedback and adaptive linear feedback.

and the following disadvantages:

- the algorithm is applicable only for digital controllers;

- the robust practical realization of the developed finite-time control scheme asks for additional computational power of the digital control device, which is required for on-line computation of the ILF value at the current state.

\section{REFERENCES}

J. Adamy and A. Flemming. Soft variable-structure controls: a survey. Automatica, 40:1821-1844, 2004.

R.E. Bellman. Dynamic Programming. Princeton University Press, Princeton, NJ., 1957. 

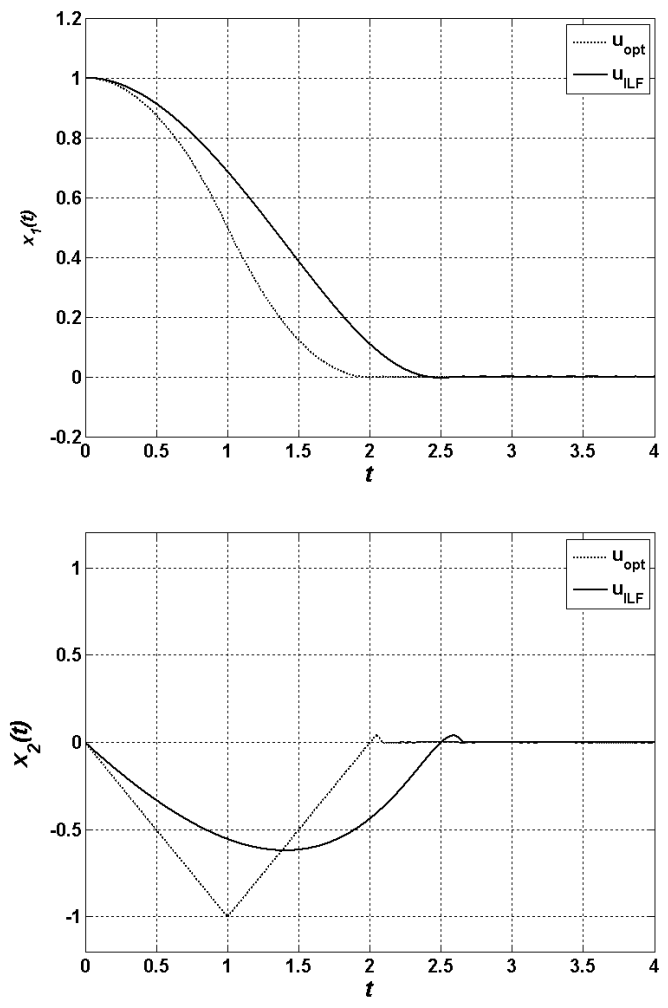

Fig. 1. The simulation results for $h=0.001$.
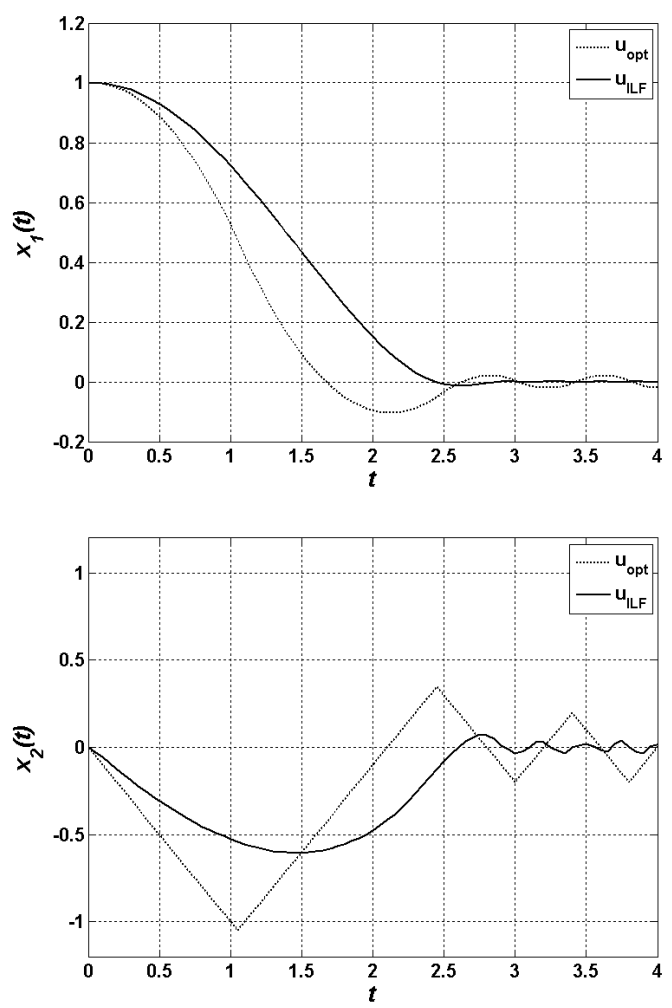

Fig. 2. The simulation results for $h=0.05$.

D. S. Bernstein. Optimal nonlinear, but continuous, feedback control of systems with saturating actuators. International Journal of Control, 62(5):1209-1216, 1995.
D.P. Bertsekas. Dynamic programming and suboptimal control: A survey from adp to mpc. European Journal of Control, 11:310-334, 2005.

S.P. Bhat and D.S. Bernstein. Finite-time stability of continuous autonomous systems. SIAM Journal of Control and Optimization, 38(3):751-766, 2000.

L. Biagiotti and R. Zanasi. Time-optimal regulation of a chain of integrators with saturated input and internal variables: An application to trajectory planning. In 8th IFAC Symposium on Nonlinear Control Systems, pages 1278-1283, 2010.

A. H. Bohl and T. J. McAvoy. Linear feedback vs. time optimal control. Industrial and Engineering Chemistry Process Design and Development, 15(1):24-33, 1976.

V. G. Boltyanski and A. Poznyak. The Robust Maximum Principle: Theory and Applications. Birkhauser, 2012.

F.L. Chernousko, I.M. Ananevskii, and S.A. Reshmin. Control of nonlinear dynamical systems: methods and applications. Berlin: Springer-Verlag, 2008.

R. Courant and F. John. Introduction to calculus and analysis (Vol. II/1). New York: Springer, 2000.

F. Dinuzzo and A. Ferrara. Higher order sliding mode controllers with optimal reaching. IEEE Transactions on Automatic Control, 54(9):2126-2136, 2009.

A. Feldbaum. Optimal processes in systems of automatic control. Automation and Remote Control (in Russian), 14(6):712-728, 1953.

V. Y. Glizer and V. Turetsky. Robust Controllability of Linear Systems. Nova Science Publishers, New York, 2012.

V.I. Korobov. A general approach to synthesis problem. Doklady Academii Nauk SSSR, 248:1051-1063, 1979.

J. B. Lasserre, C. Prieur, and D. Henrion. Nonlinear optimal control: Numerical approximations via moments and lmi-relaxations. In 44th IEEE Conference on Decision and Control, pages 1648-1653, 2005.

A. Polyakov, D. Efimov, and W. Perruquetti. Finite-time stabilization using implicit lyapunov function technique. In 9th Symposium on Nonlinear Control Systems, pages 140-145, 2013.

A. Polyakov, D. Efimov, and W. Perruquetti. Robust nonasymptotic stabilization of linear systems. International Journal of Robust and Nonlinear Control, submitted, 2014.

L.S. Pontryagin, V.G. Boltyanski, R.V. Gamkrelidze, and E.F. Mishchenko. The Mathematical Theory of Optimal Processes. Inrescience Publishers, 1962.

E. Roxin. On finite stability in control systems. Rendiconti del Circolo Matematico di Palermo, 15(3):273-283, 1966.

S. Tarbouriech and G. Garcia, editors. Control of uncertain systems with bounded inputs. Lecture notes in control and information sciences. Springer Verlag, 1997.

H.D. Tuan, P. Apkaryan, and H. Tuy. Advanced global optimization algorithms for parameterized lmis. In 38th Conference on Decision and Control, Pheonix, Arizona, USA, 1999.

V.I. Utkin, J. Guldner, and J. Shi. Sliding Mode Control in Electro-Mechanical Systems. CRC Press., 2009.

X. Wang, A. Saberi, A. Stoorvogel, and H. Fjaer. Control of a chain of integrators subject to actuator saturation and disturbances. International Journal of Robust and Nonlinear Control, 22(14):1562-1570, September 2012. 